\title{
Clinical features and predictors of multiple bronchoscopy therapy of plastic bronchitis in children
}

\author{
Tongqiang Zhang ${ }^{1}$, Lihua Zhao ${ }^{2}$, Jiafeng Zheng', Linsheng Zhao ${ }^{2}$, Xiaojian Cui², \\ Yongsheng $\mathrm{Xu}^{2}$, and Chunquan $\mathrm{Cai}^{2}$ \\ ${ }^{1}$ The children's hospital of Tianjin \\ ${ }^{2}$ Tianjin Children's Hospital
}

May 17, 2021

\begin{abstract}
Background. To analyze the clinical features of children with plastic bronchitis (PB) and identify the risk factors of multiple flexible fiberoptic bronchoscopy (FOB) therapy. Methods. Retrospective analysis was performed on 269 PB children from 2016 to 2019,144 cases were in single FOB group, 125 cases were in the multiple FOB group. The clinical manifestations, laboratory datas, imaging findings and management were investigated. The different features were compared between the single FOB group and multiple FOB group. Results. A total of 269 PB children were collected with a mean age of $6.7 \pm 2.8$ years. 257 (95.5\%) cases were diagnosed as Mycoplasma pneumonia (MP) infection. The mean duration of fever was $10.6 \pm 3.7$ days. All the patients presented with fever, and $62(23.0 \%)$ suffered from hypoxemia, 144 (53.5\%) had extrapulmonary complications. Higher levels of ESR, CRP, PCT, IL-6, LA, LDH, FER and D-dimer were observed. The proportion of pulmonary consolidation, segmental or lobar atelectasis, pleural effusion and pleural thickening were 97.4\%, 46.5\%, 47.9\% and 63.2\%, respectively. Furthermore, multivariate logistic regression analysis showed that $\mathrm{N} \%>75.5 \%, \mathrm{LDH}>598.5 \mathrm{U} / \mathrm{L}$, and D-dimmer $>0.45 \mathrm{mg} / \mathrm{L}$ were independent isk factors for multiple FOB therapy. Conclusions. MP is a significant pathogen of PB in children. Patients with PB are more likely to suffer from persistent fever, excessive inflammation and severe radiological findings. N\% $>75.5 \%, \mathrm{LDH}>598.5 \mathrm{U} / \mathrm{L}$ and D-dimmer $>0.45 \mathrm{mg} / \mathrm{L}$ may be predictors of multiple FOB treatment.
\end{abstract}

\section{Introduction}

Plastic bronchitis (PB) is an uncommon pulmonary disease characterized by formation of bronchial casts (BCs) in airways, which can partially or completely obstruct the tracheobronchial tree ${ }^{1}$. Previously, PB was usually reported in children with surgically palliated congenital heart disease, especially those after the Fontan procedure ${ }^{2}$. With the wide application of fiberoptic bronchoscopy (FOB) in bronchopulmonary disease, accumulating evidence indicated that $\mathrm{PB}$ can be triggered by common pathogens of respiratory tract infection including influenza virus (A and B), adenovirus (ADV) and Mycoplasma pneumoniae (MP) ${ }^{3-7}$, suggesting that $\mathrm{PB}$ may not be a rare disease.

The clinical manifestations of PB include repeated fever, shortness of breath and can rapidly progress to acute dyspnea and even life-threatening respiratory failure ${ }^{8,9}$. As PB is a serious disease which can endanger lives without timely management, we explored and analyzed the clinical characteristics, laboratory examinations, imaging features and management of 269 children with PB to help clinians recognize it in time and apply effective treatment promptly.To the best of our knowledge, this study is the largest research of $\mathrm{PB}$ in children and is the first study to identify risk factors of multiple FOB therapy in PB patients.

\section{Subjects and Methods}

\subsection{Study population}


We collected and analyzed the medical records and chest radiographic findings of 269 children with PB, who were admitted to Respiratory Department of Tianjin Children's Hospital from January 2016 to December 2019 .

All the cases received FOB and bronchoalveolar lavage (BAL) procedure. If the fever does not subside, and the chest X-ray does not improve 2-3 days after the first FOB operation, the patient will receive multiple FOB and BAL treatments. Subjects were divided into the single FOB group and multiple group([?]2 times) according to the times of FOB treatment. The diagnosis of PB was determined according to discovery of inflammatory BCs by FOB, and further confirmed by pathology. Hypoxemia was defined as any recorded oxygen saturation of $<92 \%$ by pulse oximetry, measured on room air ${ }^{10}$. MP infection is determined by serologic or MP polymerase chain reaction (PCR) tests. An MP-immunoglobulin M (IgM) titer [?]1:160 or four-fold rising titer in acute and convalescent serum specimens were considered positive ${ }^{11}$.

Inclusion criteria : (1) All patients had an acute onset of fever and cough. (2) All patients met the criteria of type I PB confirmed by histopathology. Exclusion criteria: (1) patients who had underlying disease, such us congenital heart disease, asthma and congenital immunodeficiency disease. (2) patients who had history of inhalation of foreign body and confirmed by FOB as bronchial foreign body. (3) Patients who had incomplete medical records.

\subsection{Methods}

The study was performed in accordance with the Declaration of Helsinki and approved by the ethics committee of the Tianjin Children's Hospital. The ethics committee waived the need for written informed consent provided by participants due to the retrospective nature of the study, because all patient data were analyzed anonymously, and no additional informed consent was required.

Clinical characteristics, laboratory findings, imaging features, and management of the 269 patients were collected at the time of admission.

Peripheral blood samples were also obtained on admission for the determination of blood routine examination, erythrocyte sedimentation rate(ESR), C-reactive protein (CRP), procalcitonin (PCT), interleukin (IL)-6, lactic acid(LA), lactic dehydrogenase (LDH), alanine aminotransferase (ALT), aspartate aminotransferase (AST), ferritin (FER), D- dimer, fibrinogen (FG) and specific antibody to MP. Blood routine examination was performed every 2-3 days and was compared between at admission and at discharge. Chest computed tomography (CT) was performed before admission or during hospitalization as these patients showed persistent fever or large infiltration on chest X-ray.

All the patients enrolled in our study received FOB, and the fluid of BAL were collected for microbiological determination according to the Guide to pediatric bronchoscopy ${ }^{12}$. Virus were identified by direct immunofluorescence PCR, MP using PCR and bacteria were detected by culture or multiplex PCR for respiratory bacteria pathogens.

The study was performed in accordance with the Declaration of Helsinki and approved by the ethics committee of the Tianjin Children's Hospital. The ethics committee waived the need for written informed consent provided by participants due to the retrospective nature of the study, because all patient data were analyzed anonymously, and no additional informed consent was required.

\subsection{Data analysis}

Datas were processed using SPSS 26.0. Continuous variables were expressed as mean \pm standard deviation (SD) or median values (interquartile range) and assessed by independent group $t$ tests or Mann-Whitney $U$ test. Categorical variables were expressed as percentage (\%) and assessed by Chi-squared tests or Fisher's exact test. Receiver Operating Characteristic (ROC) curves and Logistic regression analysis were performed to identify variables associated with multiple FOB therapy in PB patients. A two-sided $\alpha$ less than 0.05 were considered as statistically significant.

\section{Results}




\subsection{Detection rate of $\mathrm{PB}$ and its distribution in different seasons.}

Among 4958 children of pneumonia with FOB and BAL therapy, 269 subjects were diagnosed as PB from 2016 to 2019. The annual cases of PB was 30,42, 67 and 130, respectively, while the annual cases of pneumonia with FOB and BAL for adjuvant therapy was 818, 858, 1471 and 1811 . The detection rate of PB in pneumonia was $3.7 \%, 4.9 \%, 4.6 \%$ and $7.2 \%$, respectively ( 2016 to 2019) ( figure 1). The seasonal distribution of PB from 2016 to 2019 was shown in figure 2 and the peak incidence was observed in 2019 winter.

\subsection{Etiological distribution in 269 children with PB.}

In terms of etiology in the $269 \mathrm{~PB}$ children, no clear pathogen was detected in 6 cases and the positive decteciton rate was $97.8 \%$ ( 263/269), coinfections were detected in 20.4\% ( 55/269). Among single pathogen infection, MP was the most predominant pathogen $(75.1 \%, 202 / 269)$, followed by influenza virus $(0.7 \%$, 2/269), Candida albicans $(0.7 \%, 2 / 269)$, ADV $(0.4 \%, 1 / 269)$ and Streptococcus pneumoniae $(0.4 \%, 1 / 269)$. Of these 55 cases with combined infection, coinfection of MP and Streptococcus pneumoniae was the most, with a infection rate of $8.6 \%(23 / 269)$, followed by coinfection of MP and ADV (3.3\%, 9/269), MP and influenza virus $(3.3 \%, 9 / 269)$, MP and Staphylococcus $(3.0 \%, 8 / 269)$, MP and Acinetobacter baumannii $(1.5 \%, 4 / 269)$, MP and Haemophilus influenzae $(0.7 \%, 2 / 269)$. ( Table 1 )

\subsection{Clinical characteristics of $\mathrm{PB}$ in children.}

The mean age of the subjects was $6.7 \pm 2.8$ years (range, 9 months- 14 years), and the male-to-female ratio was 1.04. The mean duration of fever and hospitalization was $10.6 \pm 3.7$ and $9.3 \pm 3.2$ days, respectively. All the patients presented with cough and fever, and $62(23.0 \%)$ cases suffered from hypoxemia. Of these 269 patients, $3(1.1 \%)$ had pulmonary embolism, $15(5.6 \%)$ cases was diagnosed as necrotizing pneumonia, and $144(53.5 \%)$ had extrapulmonary complications, including 16(5.9\%) with leukopenia, 62(23\%) with digestive system abnormalities (nausea, vomiting, elevated transaminase), 24(8.9\%) with cardiovascular system abnormalities (elevated myocardial enzymes, abnormal electrocardiograph(ECG), pericardial effusion and cardiac thrombosis), 40(14.9\%) with rash, 21(7.8\%) with toxic encephalopathy, and 27(10\%) with electrolyte disorder.

Among these 269 patients, 144 cases underwent FOB and BAL procedure for once(the single group) and 125 underwent multiple therapy (the multiple group). There were no statistically differences between the two groups in age, sex ratio, incidence of fever. Compared with single group, children in the multiple group exhibited higher peak body temperature, longer duration of fever and hospitalization. The total incidence of extrapulmonary complications was higher in the multiple group, especially in digestive system, however differences in incidence of blood system, cardiovascular system, skin, central nervous system and lectrolyte disorder were not observed. ( Table 2 )

\subsection{Laboratory characteristics of PB in children.}

Laboratory indicators were summarized in Table 3. Higher levels of ESR, CRP, PCT, IL-6, LA, LDH, FER and D-dimer were observed in PB patients. The levels of N\%, CRP, IL-6, LA, ALT, AST, LDH, FER, Ddimer in multiple group were higher than those in the single group, and the differences were statistically significant ( all, $\mathrm{P}<0.05$ ). ( Table 3 )

\subsection{Imaging characteristics of $\mathrm{PB}$ in children.}

All the 269 enrolled patients underwent chest CT scan and 262 (97.4\%) showed pulmonary consolidation, 125 (46.5\%) had segmental or lobar atelectasis, $127(47.9 \%)$ with pleural effusion and170 (63.2\%) with pleural thickening. The incidence of pulmonary consolidation and pleural effusion were higher in multiple group ( $100 \%$ vs $95.1 \%, 56.8 \%$ vs $38.9 \%$, respectively, $\mathrm{P}<0.05)$. However, there was no difference in the incidence of segmental or lobar atelectasis and pleural thickening between the two groups. ( Table 4)

\subsection{Management of PB in children}


All the patients had been administrated with at least one type of antibiotics before admission. 269 subjects were prescribed anti-MP antibiotics empirically, among which 197 (73.3\%) were treated with Macrolides, $52(19.3 \%)$ with Doxycycline, $20(7.4 \%)$ with Quinolones. Combination of antibiotics, including anti-MP and anti-bacteria or anti-influenza virus were applied to $261(97 \%)$ subjects. $257(95.5 \%)$ cases received oral or intravenous glucocorticoid, 55 (20.4\%) received intravenous immunoglobulin (IVIG), 48 (17.8\%) were admitted to intensive care unit(ICU) and $35(13 \%)$ received mechanical ventilation. The multiple group exhibited a higher proportion of glucocorticoid and IVIG therapy compared to the single group (98.4\% vs $92.4 \%, 27.2 \%$ vs $14.6 \%$, respectively, $\mathrm{P}<0.05)$. FOB and BAL was used to remove mucus plug and BCs. All the patients were discharged without mortality. (Table 5)

\subsection{Risk factors for patients with multiple FOB therapy}

Univariate analysis identified peak body temperature, duration of fever and hospitalization, hypoxemia, complications of extrapulmonary, presence of pleural effusion, higher level of inflammation indicators and D-dimer as significant related factors for multiple FOB. ROC analysis revealed that N\%, LDH and D-dimer were of great significance in identifiying subjects required multiple FOB. The cutoff values for these three variables were $75.5 \%, 598.5 \mathrm{U} / \mathrm{L}$ and $0.45 \mathrm{mg} / \mathrm{L}$ with maximum sensitivities and specificities. To adjust for the influence of confounders, multivariate logistic regression analysis was performed, and results showed that $\mathrm{N} \%, \mathrm{LDH}$ and D-dimer were independent risk factors for multiple FOB with the odds ratio (OR) values of $3.777,2.729$ and 2.272 , respectively. (Table 6)

\section{Discussion}

In recent 10 years, an increasing number of studies about $\mathrm{PB}$ in children associated with respiratory infections are being reported ${ }^{3-7}$. However, many literatures on $\mathrm{PB}$ and $\mathrm{BCs}$ are composed of case reports or small case series, and accurate epidemiological data of $\mathrm{PB}$ and $\mathrm{BCs}$ are still lacking. Lu S et al. ${ }^{6}$ reported 22 cases of BCs among 161 MPP children with FOB and BAL treatment from November 2015 to December 2016. Wei $\mathrm{F}$ et $\mathrm{al}^{13}$ analyzed a study of $63 \mathrm{~PB}$ children associated with influenza virus from May 2014 to April 2020. In the study, we identified 269 children with PB from 4958 cases of pneumonia with FOB and BAL treatment, and we estimated that PB accounted for $5.4 \%$ in children with pneumonia requiring FOB.

We identified that MP, bacteria, influenza virus, ADV and Candida albicans can trigger PB. In rencent years, PB associated with MP has been reported in various studies. In a investigation enrolled 15 children with PB, MP infection accounted for $86.7 \%$ of the cases ${ }^{4}$. Guo et al. also identified that in a study of 73 subjects with type I PB, MP infection was detected in $90.4 \%$ of the children ${ }^{14}$. In the present study which is the largest research to date of PB, MP was positive in 257 ( $95.5 \%)$ patients, including single MP infecton in $202(75.1 \%)$ cases and coinfection of MP with bacteria and/ or virus in 55(20.4\%) subjects. Moreover, the seasonal distribution of PB from 2016 to 2019 indicated that the peak incidence of PB was observed in winter, especially in 2019 winter. Yan X also ${ }^{15}$ demonstrated that MPP had a higher prevalence rate in winter and peaks occurred in November 2019 in a 3-year retrospective analysis from Bei Jing. The considerable detection rate of MP in PB and epidemic consistency of PB and MPP indicated that MP is a prominent pathogen of PB. MPP is usually considered to be self-limited and benign ${ }^{16}$, however it may proceed to severe or fulminant pneumonia, endanger the lives ${ }^{17,18}$. Previous studies ${ }^{6,19,20}$ also showed that MP infection can lead to varying degrees of respiratory mucus plug, even BCs, resulting in PB. The mechanism of its role in PB maybe that MP infection not only directly cause damage to the airway, including epithelial necrosis to block the respiratory tract and cilia shedding to cause cilia removal dysfunction, but also promote airway hypersecretion by the excessive inflammation ${ }^{21,22}$. Compared with bacterial and viral infections, MP infection is more likely to induce excessive inflammatory response in the body ${ }^{23}$ which can induce continuous formation of mucus plug in the airway and cause damage to the whole body.

The mean age of our patients was $6.7 \pm 2.8$ years (range, 9 months-14 years) which was similar to the $6.1 \pm$ 2.8 years reported in previous study ${ }^{14}$. The clinical manifestations of $\mathrm{PB}$ are diverse, including fever, cough, dyspnea or respiratory distress and damage to extrapulmonary system, among which rapid progression to hypoxemia can be applied as a strong indicator of PB. However, when patients with mild symptoms have 
no or mild signs of hypoxemia, many clinicians cannot recognize it. In our study, $62(23.0 \%)$ cases suffered from hypoxemia. Li W et al. [18] revealed that in their study all the 15 children with PB showed no signs of hypoxemia, and Lu S et al. ${ }^{6}$ reported that only 9 out of 22 children with MPP BCs received oxygen therapy. All the above suggested that hypoxemia was not sensitive enough to discover PB. Therefore, we should comprehensively evaluate the clinical manifestations in order to recognize PB timely.

The incidence of ICU treatment in our study was $17.8 \%$ ( 48/269 cases), which was lower than that of $58.3 \%$ ( $14 / 24$ cases) in Lu et al's study ${ }^{24}$, and no death cases were observed in our study. The rate of critically ill and mortality was significantly lower than previous descriptions ${ }^{8,25}$. The possible explanation may be attributed to the following two aspects. On one hand, the clinical manifestation of PB depends on the location and degree of bronchial obstruction, ranging from fragmented partial BCs to a large and complete cast that fills the entire airway ${ }^{6}$. On the other hand, rapid FOB treatment contributed to early effective intervention and prevented the development of respiratory failure.

We found that patients in the multiple group exhibited severe clinical manifestations, including higher peak body temperature, longer duration of fever and hospitalization, higher incidence of intra and extra-pulmonary complications, higher levels of inflammation indicators and D-dimer. Furthermore, multiple logistic regression identified that $\mathrm{N} \%>75.5 \%, \mathrm{LDH}>598.5 \mathrm{U} / \mathrm{L}$ and $\mathrm{D}$-dimer $>0.45 \mathrm{mg} / \mathrm{l}$ were the independent risk factors for multiple FOB therapy. It was reported that higher neutrophil(63.1\%) was positively correlated with excessive inflammation and disease severity ${ }^{26}$ in children with MPP. LDH is a nonspecific inflammatory biomarker and exists within the cytoplasm. Xu et al. ${ }^{27}$ identified LDH as independent risk factor for mucus plug formation in children with RMPP and our results showed that LDH $>598.5 \mathrm{U} / \mathrm{L}$ is a predictor of multiple FOB therapy. Although the pathogenesis of $\mathrm{PB}$ was not completely clear, at present it is commonly believed that PB triggered by infection result from inappropriate immune reponse to infection and direct damage of pathogen to the airway ${ }^{3,28}$. The higher level of inflammation biomarkers indicate the excessive inflammation, which can lead to continuous formation of mucus plug, resulting in multiple FOB to clear the subsequent BCs.

The increase of D-dimer is an important indicator of high fibrinolysis, representing blood hypercoagulability and the presence of thrombi ${ }^{29}$. It was reported ${ }^{30}$ that the D-dimer level in the severe MPP group was higher than that in the mild group in children $(0.61 \mathrm{vs.} .30 \mathrm{mg} / \mathrm{L})$, and the level of D-dimer was positively correlated with the severity of MPP. In the study, we found an elevated D-dimer level in PB children and D-dimer $>0.45 \mathrm{mg} / \mathrm{l}$ was a risk factor for multiple FOB and BAL treatments, which was consistent with the view of Zhang et $\mathrm{al}^{31}$. Their study showed that children receiving multiple FOB treatments for RMPP had higher D-dimmer levels $(1.808 \mathrm{mg} / \mathrm{L})$ compared with the monotherapy group $(0.567 \mathrm{mg} / \mathrm{L})$. However, the median level of D-dimer in our study was lower than that of Zhang et al and we speculated that there are two possible explanations. On one hand, the enrolled subjects in the two study were different. RMPP children may exhibit higher D-dimmer level duo to intensive body reponse to MP infection. On the other hand, in the present study, D-dimer level of a significant number of children may not be measured at the peak of disease process. In summary, we speculated that hypercoagulability play an important role in inducing subsequent mucus plugs formation of $\mathrm{PB}$ and higher D-dimer level is an important risk factor for patients requiring multiple FOB treatments.

The imaging features of children with PB were diverse, including pulmonary consolidation, atelectasis, pleural effusion, emphysema and pneumothorax ${ }^{14,32}$. Recent literature [18] found that 13 out of 15 PB children had lung consolidation involved unilateral or bilateral infiltration, and 5 cases developed pleural effusion. Lu S et al. ${ }^{6}$ also observed that all 22 children with BCs had lobar consolidation and 6 cases developed atelectasis. Our results showed that the imaging manifestations of $\mathrm{PB}$ were not specific, and $\mathrm{PB}$ patients were more likely to be associated with lung consolidation (97.4\%), which was consistent with the $98.6 \%$ of PB children with lung consolidation or atelectasis reported previously ${ }^{14}$. Therefore, we concluded that PB should be considered when patients with persistent fever and large chest imaging infiltration.

Although PB presented with severe clinical manifestations and the critical form in children has a mortality rate as high as 7-10\% due to failing to extract BCs in time $e^{8,9,25}$, the prognosis of $\mathrm{PB}$ is generally favorable if the disease can be treated promptly. Most reports ${ }^{4,6}$ of effective therapy were based on standard antibiotic 
treatment, glucocorticoids, IVIG and clearance of BCs with FOB. In agreement with this notion, all patients in the present study received appropriate antibiotic treatment, up to $95.5 \%$ subjects received glucocorticoid therapy, and $20.4 \%$ received IVIG to modulate immunity. FOB procedure is of prominent efficacy in treatment of $\mathrm{PB}$, including direct clearance of BCs to improve lung ventilation, the clearance of various inflammatory factors and easy access to the lower airway for the pathogenic detection. Recent studies $^{33,34}$ found that compared with late FOB therapy, FOB therapy during the early disease process in RMPP patients with large pulmonary lesions resulted in faster recovery of clinical and inflammation characters and shorter hospital stay. Furthermore, there are a considerable number of children with PB requiring multiple FOB therapy. In our study, the proportion of patients in mutiple group was $46.5 \%(125 / 269)$ which was consistent with the result of Cai $\mathrm{L}^{35}$. Their study showed that more than $50 \%$ children with $\mathrm{PB}$ received multiple FOB treatment and all achieved favorable prognosis. In summary, we believed that in patients with persistent fever, higher level of inflammation indicators and large infiltration in chest imaging, FOB is of great significance in timely diagnosis and effective treatment.

There were several limitations to this study. Firstly, it was a retrospective study and there may have been some selection bias. Secondly the patients were enrolled from a single center and the results may not easily extrapolate to patients admitted to other regions. Thirdly, to timely identify PB and avoid improper application of FOB, a RCT study should be designed between PB and such diseases.

\section{Conclusion}

In conclusion, our study showes that MP is a significant pathogen of PB. The clinical manifestations of PB are not specific, children with $\mathrm{PB}$ might be easier to suffer from persistent fever, excessive inflammation and severe radiological findings. $\mathrm{N} \%>75.5 \%, \mathrm{LDH}>598.5 \mathrm{U} / \mathrm{L}$ and D-dimer $>0.45 \mathrm{mg} / \mathrm{L}$ are important risk factors for multiple FOB procedures. Favorable prognosis can be expected with timely diagnosis and appropriate FOB treatment.

Acknowledgments. The authors wish to thank the patients and participating investigators and staff associatied with the cilinical studies discussed here.

Conflict of interest. The authors have no Conflicts of interest to declare.

Availability of data and materials. The datasets used and/or analysed during the current study are available from the corresponding author on reasonable request.

\section{References}

1. Walker PA, Shah SK, Letourneau PA, Allison ND, Cox CS. Treatment of plastic bronchitis using serial flexible bronchoscopy and aerosolized heparin therapy. Eur J Pediatr Surg. 2013;23(2):157-160.

2. Caruthers RL, Kempa M, Loo A, et al. Demographic characteristics and estimated prevalence of Fontanassociated plastic bronchitis.Pediatr Cardiol. 2013;34(2):256-261.

3. Yuan L, Huang JJ, Zhu QG, Li MZ, Zhuo ZQ. Plastic bronchitis associated with adenovirus serotype 7 in children. BMC Pediatr.2020;20(1):268.

4. Wang L, Wang W, Sun J, et al. Efficacy of fiberoptic bronchoscopy and bronchoalveolar lavage in childhoodonset, complicated plastic bronchitis. Pediatric pulmonology. 2020.

5. Lu Z, Zheng Y. Plastic bronchitis associated with adenovirus infection. Lancet Infect Dis. 2018;18(4):474.

6. Lu S, Liu J, Cai Z, Shuai J, Huang K, Cao L. Bronchial casts associated with Mycoplasma pneumoniae pneumonia in children. Journal of International Medical Research. 2020;48(4).

7. Krenke K, Krenke R, Krauze A, Lange J, Kulus M. Plastic bronchitis: an unusual cause of atelectasis. Respiration. 2010;80(2):146-147.

8. Kunder R, Kunder C, Sun HY, et al. Pediatric plastic bronchitis: case report and retrospective comparative analysis of epidemiology and pathology. Case Rep Pulmonol. 2013;2013:649365. 
9. Cai X, Sun J, Li W, Cheng H. Clinical analysis of severe plastic bronchitis in 8 children. Zhonghua Wei Zhong Bing Ji Jiu Yi Xue.2016;28(1):73-75.

10. Izumikawa K, Izumikawa K, Takazono T, et al. Clinical features, risk factors and treatment of fulminant Mycoplasma pneumoniae pneumonia: a review of the Japanese literature. J Infect Chemother.2014;20(3):181185.

11. Lee SC, Youn YS, Rhim JW, Kang JH, Lee KY. Early Serologic Diagnosis of Mycoplasma pneumoniae Pneumonia: An Observational Study on Changes in Titers of Specific-IgM Antibodies and Cold Agglutinins. Medicine (Baltimore). 2016;95(19):e3605.

12. Pediatric Bronchoscopy Collaborative Group TSGoRDTSoPCMA. Guide to pediatric bronchoscopy (2009 edition). Zhonghua Er Ke Za Zhi.2009;47(10):740-744.

13. Wei F, Wen FQ, Yang WG, Zheng YJ. Clinical features of children with influenza and plastic bronchitis: an analysis of 63 cases.Zhongguo Dang Dai Er Ke Za Zhi. 2020;22(10):1105-1108.

14. Guo YS ZY, Zhai J, Zhao L, Shen Y, Guo R, Han B. Clinical analysis of 73 children with type I plastic bronchitis. Journal of Tianjin Medical University. 2017;23(5):422-425.

15. Xing Y, Wang D, Sheng K, et al. Dynamic change of Mycoplasma pneumoniae pneumonia in hospitalized children in a general hospital: a 3-year retrospective analysis. Transl Pediatr. 2020;9(4):522-531.

16. Meyer Sauteur PM, Krautter S, Ambroggio L, et al. Improved Diagnostics Help to Identify Clinical Features and Biomarkers That Predict Mycoplasma pneumoniae Community-acquired Pneumonia in Children. Clin Infect Dis. 2020;71(7):1645-1654.

17. Takiguchi Y, Shikama N, Aotsuka N, Koseki H, Terano T, Hirai A. Fulminant Mycoplasma pneumoniae pneumonia. Intern Med.2001;40(4):345-348.

18. Wang X, Zhong LJ, Chen ZM, Zhou YL, Ye B, Zhang YY. Necrotizing pneumonia caused by refractory Mycoplasma pneumonia pneumonia in children. World J Pediatr. 2018;14(4):344-349.

19. Ling Y, Zhang T, Guo W, et al. Identify clinical factors related to Mycoplasma pneumoniae pneumonia with hypoxia in children. BMC Infectious Diseases. 2020;20(1).

20. Wang L, Lu S, Feng Z, et al. The early examination of combined serum and imaging data under flexible fiberoptic bronchoscopy as a novel predictor for refractory Mycoplasma pneumoniae pneumonia diagnosis. Medicine (Baltimore). 2017;96(50):e9364.

21. Liang H, Jiang W, Han Q, Liu F, Zhao D. Ciliary ultrastructural abnormalities in Mycoplasma pneumoniae pneumonia in 22 pediatric patients. Eur J Pediatr. 2012;171(3):559-563.

22. Moser C, Nussbaum E, Cooper DM. Plastic bronchitis and the role of bronchoscopy in the acute chest syndrome of sickle cell disease. Chest. 2001;120(2):608-613.

23. Jiao AX MY, Rao XC, Pan YN, Hu YH, Jiang QB:. Plastic bronchitis caused by mycoplasma pneumoniae pneumonia and bacterial pneumonia in children:15 cases clinical analysis. Chin J Evid Based Pedia$\operatorname{tr} 2010 ; 5(4): 294-298$.

24. Lu ZW DJ, Zeng YJ, Wang L, Ma HJ, L J. Clinical analysis of 24 children with plastic bronchitis. Chin J Appl Clin Pediatr.2013;28(4):265-267.

25. Brogan TV, Finn LS, Pyskaty DJ, Jr., et al. Plastic bronchitis in children: a case series and review of the medical literature.Pediatr Pulmonol. 2002;34(6):482-487.

26. Wang M, Wang Y, Yan Y, et al. Clinical and laboratory profiles of refractory Mycoplasma pneumoniae pneumonia in children. Int $J$ Infect Dis. 2014;29:18-23. 
27. Xu Q, Zhang L, Hao C, et al. Prediction of Bronchial Mucus Plugs Formation in Patients with Refractory Mycoplasma Pneumoniae Pneumonia.J Trop Pediatr. 2017;63(2):148-154.

28. Zhang FZ, Qin L, Yuan JX, Tang LF. Plastic bronchitis due to adenoviral infection: a case report. BMC Pediatr. 2020;20(1):61.

29. Li T, Yu H, Hou W, Li Z, Han C, Wang L. Evaluation of variation in coagulation among children with Mycoplasma pneumoniae pneumonia: a case-control study. J Int Med Res. 2017;45(6):2110-2118.

30. Jin X, Zhu Y, Zhang Y, Chen J, Rong L, Zhao X. Assessment of levels of D-dimer and interferongamma in pediatric patients with Mycoplasma pneumoniae pneumonia and its clinical implication. Exp Ther Med.2018;16(6):5025-5030.

31. Zhang R W, Jiang WJ, Wang MJ, Chen ZR, Huang L, Zhu CH, Ji W, YanYD, Wang YQ, Hao CL. Risk factors of multiple bronchoscope lavage therapy in children with refractory Mycoplasma pneumoniae pneumonia. Chin J Appl Clin Pediatr. 2018;5(4):1694-1698.

32. Li XL GW, Dong HQ, Ning J, Ren LX, Xu YS, Wan LY, Liu FJ. Clinical analysis of 30 children with plastic bronchitis. Guangxi Medical Journal. 2016;38(9):1239-1241.

33. Soyer T, Yalcin S, Emiralioglu N, et al. Use of serial rigid bronchoscopy in the treatment of plastic bronchitis in children. J Pediatr Surg. 2016;51(10):1640-1643.

34. Su DQ, Li JF, Zhuo ZQ. Clinical Analysis of 122 Cases with Mycoplasma Pneumonia Complicated with Atelectasis: A Retrospective Study. Adv Ther. 2020;37(1):265-271.

35. Cai LH, Li SS, Qu CY, Yan YD, Wang MJ, Ji W. Clinical characteristics of plastic bronchitis after pneumonia in children and the value of bronchoscopy indiagnosis and treatment. Chin J Apl Clin Pediatr. 2020;35(21):1638-1642.

\section{Hosted file}

table.pdf available at https://authorea.com/users/414147/articles/522273-clinical-featuresand-predictors-of-multiple-bronchoscopy-therapy-of-plastic-bronchitis-in-children

Figure 1 The annual case number of PB from 2016 to 2019

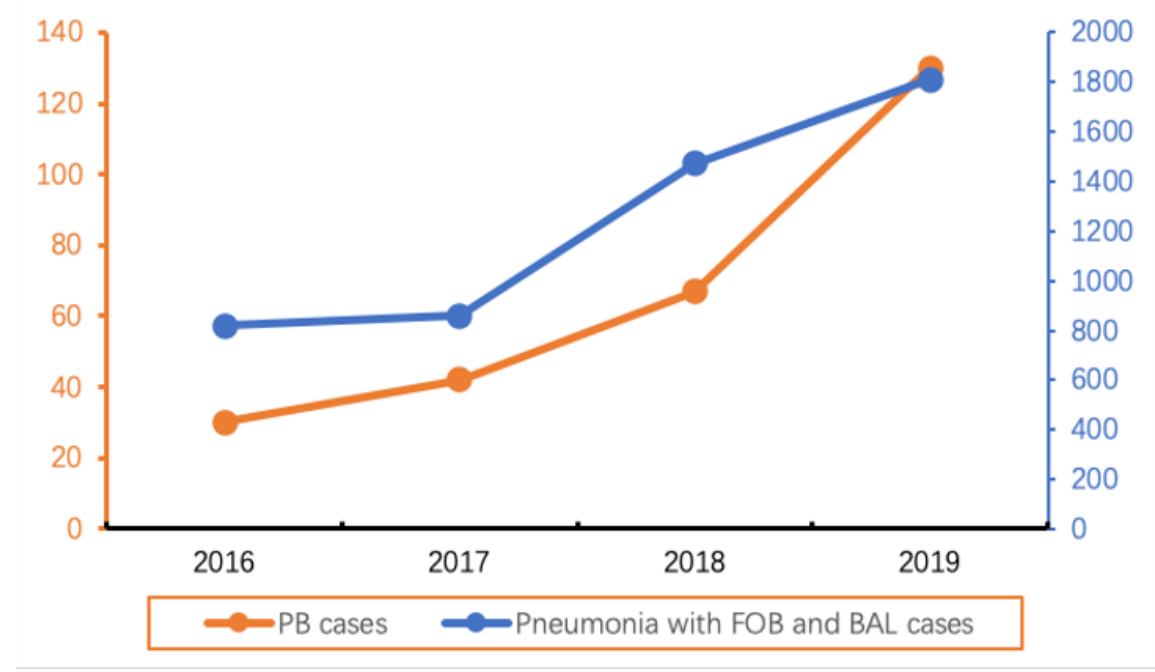


Figure 2 Seasonal distribution of PB from 2016 to 2019

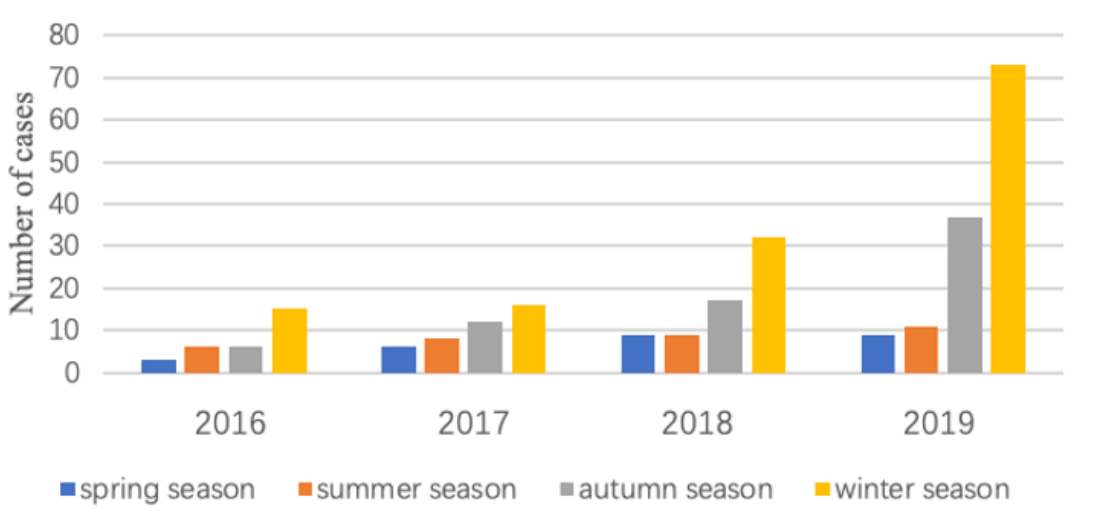

